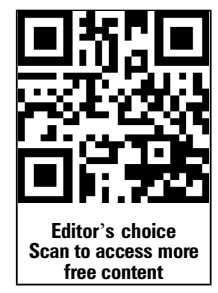

ORIGINAL ARTICLE

\title{
Significant lead-induced tricuspid regurgitation is associated with poor prognosis at long-term follow-up
}

\author{
Ulas Höke, ${ }^{1,2}$ Dominique Auger, ${ }^{1}$ Joep Thijssen, ${ }^{1}$ Ron Wolterbeek, ${ }^{3}$ \\ Enno T van der Velde, ${ }^{1}$ Eduard R Holman, ${ }^{1}$ Martin J Schalij, ${ }^{1}$ Jeroen J Bax, ${ }^{1}$ \\ Victoria Delgado, ${ }^{1}$ Nina Ajmone Marsan ${ }^{1}$
}

\begin{abstract}
- Additional material is published online only. To view please visit the journal online (http://dx.doi.org/10.1136/ heartjnl-2013-304673).

${ }^{1}$ Department of Cardiology, Leiden University Medical Centre, Leiden, The Netherlands

${ }^{2}$ Interuniversity Cardiology Institute of The Netherlands, Utrecht, The Netherlands ${ }^{3}$ Department of Biostatistics, Leiden University Medical

Centre, Leiden, The Netherlands
\end{abstract}

\section{Correspondence to} Dr Nina Ajmone Marsan, Department of Cardiology, Leiden University Medical Centre, Albinusdreef 2, Leiden 2333 ZA, The Netherlands; N.Ajmone@lumc.nl

Received 17 July 2013 Revised 26 November 2013 Accepted 20 December 2013 Published Online First 21 January 2014

\section{Linked}

- http://dx.doi.org/10.1136/ heartjnl-2013-305459

To cite: Höke $U$, Auger $D$, Thijssen J, et al. Heart 2014;100:960-968.

\begin{abstract}
Background Although the presence of an RV lead is a potential cause of tricuspid regurgitation (TR), the clinical impact of significant lead-induced TR is unknown.

Objective To evaluate the effect of significant leadinduced TR on cardiac performance and long-term outcome after cardioverter-defibrillator (ICD) or pacemaker implantation.
\end{abstract}

Methods A retrospective cohort of 239 ICD ( $n=191)$ or pacemaker $(n=48)$ recipients (age $60 \pm 14$ years, $77 \%$ male) from a tertiary care university hospital, with an echocardiographic evaluation before and within 1-1.5 years after device implantation were included. Significant lead-induced TR was defined as TR worsening, reaching a grade $\geq 2$ at follow-up echocardiography. During long-term follow-up (median 58, IQR 35-76 months), all-cause mortality and heart failure related events were recorded.

Results Before device implantation, most patients had TR grade 1 or $2(64.0 \%)$ or no TR (33.9\%), but after lead placement, significant TR was seen in 91 patients (38\%). Changes in cardiac volumes and function at follow-up were similar between patients with and without significant lead-induced TR, except for larger RV diastolic area $\left(17 \pm 6 \mathrm{~mm}^{2}\right.$ vs $\left.16 \pm 5 \mathrm{~mm}^{2}, p=0.009\right)$, larger right atrial diameter $(39 \pm 10 \mathrm{~mm}$ vs $36 \pm 8 \mathrm{~mm}$, $p<0.001)$ and higher pulmonary arterial pressures $(41 \pm 15 \mathrm{~mm} \mathrm{Hg}$ vs $33 \pm 10 \mathrm{~mm} \mathrm{Hg}, p<0.001)$ in patients with significant lead-induced TR. Patients with significant lead-induced TR had worse long-term survival ( $H R=1.687, p=0.040)$ and/or more heart failure related events $(H R=1.641, p=0.019)$. At multivariate analysis, significant lead-induced TR was independently associated with all-cause mortality $(H R=1.749, p=0.047)$ together with age, LVEF and percentage RV pacing.

Conclusions Significant lead-induced TR is associated with poor long-term prognosis.

\section{INTRODUCTION}

Trivial tricuspid regurgitation (TR) is a common echocardiographic finding in healthy individuals. ${ }^{1}$ However, significant TR (grade $\geq 2$ ) has been shown to be associated with poor prognosis, regardless of the underlying cardiac pathology. ${ }^{2}$ Significant TR may be a primary valvular disease (due to valve lesion) or secondary to tricuspid annular dilatation and/or RV remodelling. In addition, placement of an RV (trans-tricuspid) lead has also been associated with a higher risk of TR. However, the incidence of lead-induced TR, time course and effects on long-term outcome remain unknown. $^{3-8}$ Previous studies have reported the incidence of TR immediately after implantation, focusing on the potential mechanisms of valve dysfunction (perforation, impingement, adherence to the leaflets). ${ }^{4}{ }^{9}$ However, data on the long-term incidence of TR after device implantation and, more importantly, data on the impact of significant TR on cardiac performance and clinical outcome, are still lacking. Increasing treatment with devices, with growing numbers of implanted pacemakers (PMs) and cardioverter-defibrillators (ICDs), and ageing of the population may result in an increased incidence of lead-induced TR, with important clinical consequences. ${ }^{10-13}$ Therefore, the objective of this evaluation was first, to assess the incidence of significant lead-induced TR at long-term follow-up and second, to evaluate the impact of significant lead-induced TR on cardiac performance and on long-term prognosis.

\section{PATIENTS AND METHODS \\ Patients}

Patients undergoing an ICD or PM implantation at Leiden University Medical Center between January 2002 and June 2009 were included in this analysis. Data on baseline patient characteristics, implantation procedure, device characteristics and settings, and all follow-up visits were prospectively collected in the departmental cardiology information system (EPD-Vision, Leiden University Medical Center, Leiden, the Netherlands) and retrospectively analysed. Indication for device implantation, based on international guidelines, was primary and secondary prevention of sudden cardiac death in ICD recipients, and sick sinus syndrome and advanced atrioventricular block in PM recipients. ${ }^{13}$ Owing to evolving guidelines, particularly for prevention of sudden cardiac death, eligibility for device implantation in this population might have changed over time, based on the results of landmark clinical trials. $^{13-15}$

Patients with de novo implantation of pacing devices were included. Exclusion criteria were (1) previous transvenous (temporary) cardiac pacing system implantation, cardiac valve surgery, 
Table 1 Baseline clinical and echocardiographic characteristics of the patient population

\begin{tabular}{|c|c|c|c|c|}
\hline Characteristics & $\begin{array}{l}\text { Overall } \\
(n=239)\end{array}$ & $\begin{array}{l}\text { No significant lead-induced TR } \\
(\mathrm{n}=148)\end{array}$ & $\begin{array}{l}\text { Significant lead-induced TR } \\
(\mathrm{n}=91)\end{array}$ & p Value \\
\hline Age, years & $60 \pm 14$ & $60 \pm 14$ & $61 \pm 13$ & 0.893 \\
\hline Male, $\mathrm{n}(\%)$ & $184(77)$ & $114(77)$ & $70(77)$ & 0.985 \\
\hline Ischemic heart disease, $\mathrm{n}(\%)$ & $153(64)$ & $96(65)$ & $57(63)$ & 0.728 \\
\hline QRS duration, ms & $114 \pm 28$ & $112 \pm 28$ & $116 \pm 28$ & 0.362 \\
\hline $\mathrm{PM} / \mathrm{ICD}, \mathrm{n}(\%)$ & $48(20) / 191(80)$ & $29(20) / 119(80)$ & $19(21) / 72(79)$ & 0.810 \\
\hline PM indication: SSS/AV block, n (\%) & $27(56) / 21(44)^{*}$ & $16(55) / 13(45)^{*}$ & $11(58) / 8(42)^{*}$ & 0.955 \\
\hline ICD indication: primary prevention $\mathrm{n}(\%)$ & $119(62) \dagger$ & $78(66) \dagger$ & $41(57) \dagger$ & 0.183 \\
\hline Percentage of pacing for PM, median [IQR] & $100[100-100]$ & 100 [100-100] & $100[97-100]$ & 0.065 \\
\hline Percentage of pacing for ICD, median [IQR] & $0[0-1]$ & $0[0-0]$ & $0[0-2]$ & 0.149 \\
\hline Atrial fibrillation, $\mathrm{n}(\%)$ & $75(31)$ & $40(27)$ & $35(38)$ & 0.056 \\
\hline Diabetes, n (\%) & $42(18)$ & $29(20)$ & $13(14)$ & 0.308 \\
\hline NYHA functional class & $2[1-2]$ & $2[1-2]$ & $2[1-2]$ & 0.727 \\
\hline LVEDV, mL & $151 \pm 63$ & $149 \pm 58$ & $151 \pm 71$ & 0.808 \\
\hline LVESV, mL & $95 \pm 54$ & $96 \pm 49$ & $95 \pm 61$ & 0.892 \\
\hline LVEF, \% & $39 \pm 14$ & $38 \pm 14$ & $40 \pm 13$ & 0.356 \\
\hline E/A ratio & $1.0[0.7-1.4]$ & $1.1[0.8-1.6]$ & $1.0[0.7-1.4]$ & 0.748 \\
\hline E-wave deceleration time, ms & $225 \pm 74$ & $217 \pm 73$ & $238 \pm 75$ & 0.053 \\
\hline Average $\mathrm{E}^{\prime}, \mathrm{cm} / \mathrm{s}$ & $6.49 \pm 2.41$ & $6.22 \pm 2.16$ & $6.93 \pm 2.72$ & 0.096 \\
\hline E/E' ratio & 11 [8-15] & $11[8-15]$ & $12[8-16]$ & 0.737 \\
\hline Mitral regurgitation grade $\geq 2 \ddagger$ & $62(29)$ & $40(29)$ & $22(27)$ & 0.723 \\
\hline Left atrial volume, $\mathrm{mL} / \mathrm{m}^{2}$ & $36 \pm 7$ & $36 \pm 7$ & $37 \pm 8$ & 0.443 \\
\hline $\mathrm{RV}$ end-diastolic area, $\mathrm{mm}^{2}$ & $16.1 \pm 5.0$ & $16.5 \pm 5.1$ & $15.3 \pm 4.8$ & 0.074 \\
\hline RV fractional area change, $\%$ & $39 \pm 12$ & $38 \pm 13$ & $41 \pm 11$ & 0.082 \\
\hline TAPSE, mm & $17.0 \pm 4$ & $17.0 \pm 4.4$ & $17.4 \pm 4.5$ & 0.481 \\
\hline Right atrial diameter, $\mathrm{cm}$ & $3.5 \pm 0.9$ & $3.6 \pm 0.9$ & $3.5 \pm 0.9$ & 0.298 \\
\hline Tricuspid annular diameter, $\mathrm{cm}$ & $3.6 \pm 0.8$ & $3.6 \pm 0.8$ & $3.6 \pm 0.8$ & 0.931 \\
\hline sPAP, $\mathrm{mm} \mathrm{Hg}$ & $33 \pm 12$ & $32.9 \pm 12.0$ & $33.0 \pm 11.0$ & 0.916 \\
\hline Tricuspid regurgitation grade 0 & 81 (33.9) & $57(38.5)$ & $24(26.4)$ & 0.056 \\
\hline Tricuspid regurgitation grade 1 & $131(54.8)$ & $71(48.0)$ & $60(65.9)$ & \\
\hline Tricuspid regurgitation grade 2 & $22(9.2)$ & $16(10.8)$ & $6(6.6)$ & \\
\hline Tricuspid regurgitation grade 3 & $5(2.1)$ & $4(2.7)$ & $1(1.1)$ & \\
\hline
\end{tabular}

Values are mean \pm SD or median [IQR].

*Among patients with PM; tamong patients with ICD; ¥MR grade was available in 217 patients.

AV block, atrioventricular block; ICD, implantable cardioverter-defibrillator; LVEDV, left ventricular end-diastolic volume; LVEF, left ventricular EF; LVESV, left ventricular end-systolic volume; NYHA, New York Heart Association; PM, permanent pacemaker; SPAP, systolic pulmonary arterial pressure; SSS, sick sinus syndrome; TAPSE, tricuspid annular plane systolic excursion; TR, tricuspid regurgitation.

congenital heart disease or organic TR, in order to exclude other causes of TR before device implantation; (2) absence of an echocardiographic evaluation within 6 months before device implantation, in order to allow appropriate comparison of implantations before and after evaluations; (3) an echocardiographic evaluation only in the first 6 months after the procedure (mainly in relation to procedure-related complications) or only more than 1.5 years after implantation (evaluation mainly driven by a new clinical event), in order to avoid selection bias; (4) occurrence of heart failure hospitalisation or other major cardiac events in the period between the two echocardiographic evaluations, in order to exclude potential confounding factors in the comparison of TR before and after device implantation; (5) upgrades of systems to cardiac resynchronisation therapy (with or without ICD capabilities), to avoid the potential beneficial effect of resynchronisation on cardiac performance. To evaluate whether lead placement might have induced significant $\mathrm{TR}$, and in order to ensure sufficient time for potential lead-related structural or functional changes to occur, only patients with a follow-up echocardiographic evaluation within 1-1.5 years after the implantation (according to standard follow-up visits) and with a minimal follow-up of 1 year after the echocardiographic evaluation were included.

\section{Device implantation, settings and interrogations}

All pacing and defibrillator systems were transvenously implanted and in all patients the RV lead was implanted in the RV apex. The PM settings were individually tailored based on the indication for cardiac stimulation. All patients were followed up every 3-6 months after implantation and devices were interrogated at the implanting centre. To evaluate the potential confounding effect of pacing on outcome, the last percentage of pacing before follow-up echocardiography was used.

\section{Echocardiographic evaluation}

Echocardiographic assessment was made with patients in the left lateral decubitus position, using a commercially available system (Vivid 7 and E9, GE-Vingmed Ultrasound, Horton, Norway). Standard 2D and Doppler images were recorded and saved in cine-loop format for off-line analysis (EchoPac, V.110.0.0, GE-Vingmed, Horton, Norway). Echocardiographic evaluation was performed according to the most recent recommendations and included quantification of LV end-diastolic and end-systolic volumes and of LVEF by biplane Simpson's method. ${ }^{16}{ }^{17} \mathrm{LV}$ diastolic function was evaluated according to current recommendations, using transmitral flow Doppler velocities and tissue Doppler imaging-derived mitral annular velocities. ${ }^{18}$ Transmitral 
Figure 1 Distribution of tricuspid regurgitation (TR) grade in the study population before (left bars in dark grey) and after (right hand bars in lighter grey) RV lead implantation.

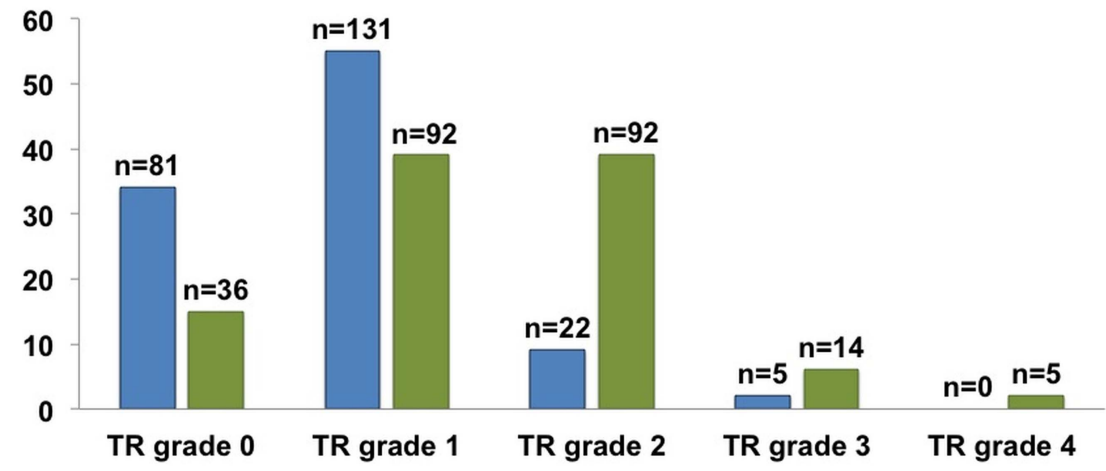

early (E) and late (A) diastolic velocities and E-wave deceleration time were measured using pulsed-wave Doppler recordings at the apical four-chamber view with a $2 \mathrm{~mm}$ sample volume at the tips of the mitral leaflets. Peak early diastolic myocardial velocities at septal and lateral borders of the mitral annulus were measured by tissue Doppler imaging and averaged to calculate the mean early diastolic myocardial velocities $\left(\mathrm{E}^{\prime}\right)$. The $\mathrm{E} / \mathrm{E}^{\prime}$ ratio was therefore derived as a measure of LV filling pressures. Mitral regurgitation severity was graded according to a multiparametric approach, as recommended. ${ }^{16}$ In addition, left atrial volume was measured by Simpson's method and indexed to body surface area. The RV dimension was assessed by tricuspid annular diameter and RV end-diastolic area, while RV function was quantified by RV fractional area change and tricuspid annular plane systolic excursion (TAPSE). ${ }^{17}{ }^{19}$ Right atrial (RA) diameter was also measured and RA pressure was estimated using the inferior vena cava size and collapsibility. Systolic pulmonary arterial pressure was estimated as the sum of the RA pressure and the peak pressure gradient between the right ventricle and right atrium, as measured on the TR spectral continuous-wave Doppler signal. ${ }^{19}$

TR severity was graded by a multiparametric approach, including assessment of the vena contracta width and regurgitant jet area by colour Doppler, evaluation of TR continuouswave Doppler signal intensity and pattern of the systolic blood flow in the hepatic veins. ${ }^{16} 19$

\section{Definition of significant lead-induced TR}

In order to evaluate the presence and impact of a significant lead-induced TR, patients with stable TR, improved TR or clinically irrelevant deterioration of TR (grade 0 or 1 ) at 1-1.5 years after implantation (no significant lead-induced TR)

Table 2 Changes in echocardiographic variables over time (from baseline to 1-1.5 year follow-up) in patients with and without significant lead-induced TR

\begin{tabular}{|c|c|c|c|c|c|}
\hline \multirow[b]{2}{*}{ Variables } & \multicolumn{2}{|c|}{$\begin{array}{l}\text { No significant lead-induced TR } \\
(\mathrm{n}=148)\end{array}$} & \multicolumn{2}{|c|}{ Significant lead-induced TR $(n=91)$} & \multirow{2}{*}{$\begin{array}{l}\mathrm{p} \text { Value } \\
\text { Interaction group and time }\end{array}$} \\
\hline & Baseline & Follow-up & Baseline & Follow-up & \\
\hline LVEDV, mL & $149 \pm 58$ & $156 \pm 65$ & $151 \pm 71$ & $163 \pm 65^{*}$ & 0.507 \\
\hline LVESV, mL & $96 \pm 49$ & $103 \pm 59^{*}$ & $95 \pm 61$ & $107 \pm 56^{*}$ & 0.441 \\
\hline LVEF, $\%$ & $38 \pm 14$ & $37 \pm 12$ & $40 \pm 13$ & $36 \pm 11^{*}$ & 0.064 \\
\hline E/A ratio & $1.1[0.8-1.6]$ & $0.9[0.7-1.4]$ & $1.0[0.7-1.4]$ & $1.1[0.6-1.9]$ & 0.961 \\
\hline E-wave deceleration time, ms & $217 \pm 73$ & $234 \pm 72$ & $238 \pm 75$ & $238 \pm 87$ & 0.227 \\
\hline Average $\mathrm{E}^{\prime}(\mathrm{cm} / \mathrm{s})$ & $6.22 \pm 2.16$ & $5.93 \pm 1.91$ & $6.93 \pm 2.72$ & $6.95 \pm 2.70$ & 0.680 \\
\hline E/E' ratio & $11[8-15]$ & 11 [8-19] & 12 [8-16] & $11[8-18]$ & 0.603 \\
\hline Mitral regurgitation grade $0 \dagger$ & $51(38)$ & $44(32)$ & $30(37)$ & $27(33)$ & 0.276 \\
\hline Mitral regurgitation grade 1 & $45(33)$ & $48(35)$ & $29(36)$ & $30(37)$ & \\
\hline Mitral regurgitation grade 2 & $31(23)$ & $33(24)$ & $16(20)$ & $18(22)$ & \\
\hline Mitral regurgitation grade 3 & $7(5)$ & $10(7)$ & $6(7)$ & $6(7)$ & \\
\hline Mitral regurgitation grade 4 & $2(2)$ & $3(2)$ & - & $1(1)$ & \\
\hline Left atrial volume $\left(\mathrm{mL} / \mathrm{m}^{2}\right)$ & $36 \pm 7$ & $38 \pm 7$ & $37 \pm 8$ & $40 \pm 9$ & 0.366 \\
\hline $\mathrm{RV}$ end-diastolic area, $\mathrm{mm}^{2}$ & $17 \pm 5$ & $16 \pm 5$ & $15 \pm 5$ & $17 \pm 6^{*}$ & 0.009 \\
\hline RV fractional area change, $\%$ & $38 \pm 13$ & $37 \pm 11$ & $41 \pm 11$ & $37 \pm 13^{*}$ & 0.154 \\
\hline TAPSE, mm & $17 \pm 4$ & $16 \pm 4^{*}$ & $17 \pm 5$ & $17 \pm 5$ & 0.849 \\
\hline Right atrium diameter, mm & $36 \pm 9$ & $36 \pm 8$ & $35 \pm 9$ & $39 \pm 10^{*}$ & $<0.001$ \\
\hline Tricuspid annular diameter, mm & $36 \pm 8$ & $36 \pm 8$ & $36 \pm 8$ & $39 \pm 9^{*}$ & 0.074 \\
\hline sPAP, mmHg & $33 \pm 12$ & $33 \pm 10$ & $33 \pm 11$ & $41 \pm 15^{*}$ & $<0.001$ \\
\hline
\end{tabular}

Values are mean \pm SD or median $[\mathrm{IQR}]$. Bold $\mathrm{p}$ values are statistically significant.

${ }^{*} p<0.05$, baseline versus follow-up.

tMitral regurgitation grade was available in 217 patients at baseline and in 220 patients at follow-up.

LVEDV, left ventricular end-diastolic volume; LVEF, left ventricular EF; LVESV, left ventricular end-systolic volume; sPAP, systolic pulmonary arterial pressure; TAPSE, tricuspid annular plane systolic excursion; TR, tricuspid regurgitation. 
were compared with patients with significant TR increase at follow-up reaching grade $\geq 2$ (significant lead-induced TR).

\section{Long-term follow-up and endpoints}

Long-term follow-up was performed by chart review and telephone contact with the general practitioner. Survival data were obtained by reviewing medical records and retrieval of survival status through the municipal civil registries. The primary endpoint was all-cause mortality. The secondary endpoint was defined as the combined endpoint of all-cause mortality and heart failure related events-that is, hospitalisation for heart failure, surgical left ventricle restoration, surgical tricuspid valvuloplasty or upgrade to cardiac resynchronisation therapy (whichever comes first).

\section{Statistical analysis}

Variables are presented as mean values $\pm S D$ when normally distributed, as median and IQR when non-normally distributed or as frequencies and percentages when variables were categorical or ordinal. Differences in baseline characteristics between the two groups were evaluated using the unpaired Student $t$ test (continuous variables) and $\chi^{2}$ (categorical data) and Wilcoxon rank sum tests (non-normally distributed continuous variables), as appropriate. Wilcoxon matched-pairs signed-rank test was used to test the significance change in the ordinal variables at follow-up. Differences in echocardiographic variables within and between the patient groups were compared by repeatedmeasures analysis of variance, including interaction between group and time. Generalised estimating equations were used to compare changes in non-normally distributed echocardiographic

A

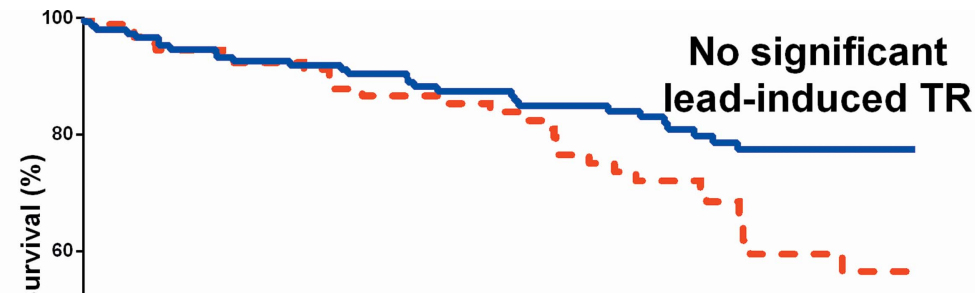

Significant lead-induced TR

音

$40-$

log rank

20- Chi square:

4.299

$p=0.038$

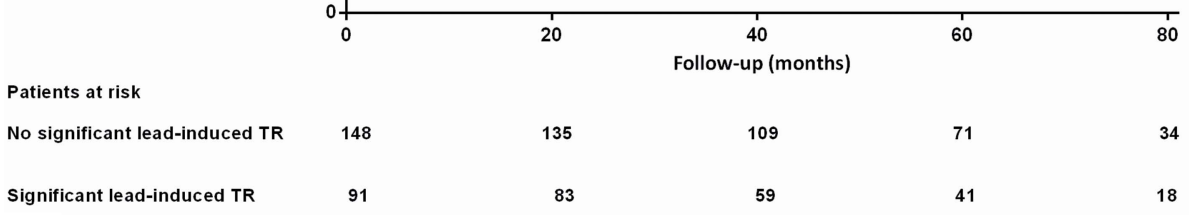

B

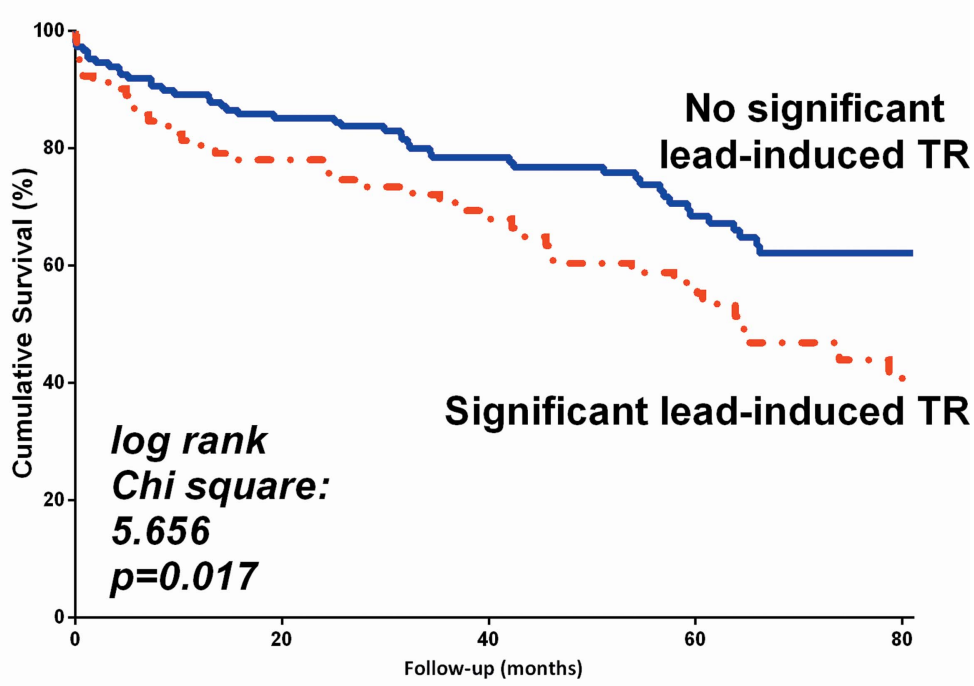

Patients at risk

No significant lead-induced TR

Significant lead-induced TR

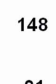

91
124

70
98

47

$\begin{array}{ll}60 & 27 \\ 32 & 13\end{array}$

Figure 2 (A) Kaplan-Meier survival curves for the time to the primary endpoint (all-cause mortality) in patients with and without significant lead-induced tricuspid regurgitation (TR) with the follow-up onset at time of the follow-up echocardiography. (B) Kaplan-Meier survival curves for the time to the secondary endpoint (all-cause mortality and heart failure related events) in patients with and without significant lead-induced TR with the follow-up onset at time of the follow-up echocardiography. 
parameters or ordinal echocardiographic parameters. Cumulative incidences with $95 \%$ CI of all-cause mortality and heart failure related events were analysed using the Kaplan -Meier method, with log-rank tests for comparison between groups. The follow-up onset was set at the moment of the follow-up echocardiographic evaluation. In addition, in patients with $\mathrm{LVEF}<40 \%$ at baseline, a subgroup analysis was performed to evaluate the impact of significant lead-induced TR on the primary and secondary endpoints. To assess whether significant lead-induced TR was associated with increased mortality and/or heart failure related events, Cox proportional hazards modelling was used. Univariate analysis was performed among clinical and echocardiographic variables at the time of the follow-up echocardiography and subsequently, all variables with a $\mathrm{p}$ value of $<0.05$ and no similarity to other parameters (in left and right ventricle dimension and function parameters), were included in the multivariable model. A $p$ value of $<0.05$ was considered statistically significant. All statistical analyses were performed using IBM PASW Statistics, V.20.0 (SPSS Inc, Chicago, Illinois, USA).

\section{RESULTS}

\section{Patients}

A total of 239 patients (184 male, mean age $60 \pm 14$ years, 191 ICD devices) were included in the present analysis. Clinical and echocardiographic characteristics of the patient population before implantation are summarised in table 1 . Indication for ICD was primary prevention in 119/191 (62\%) patients, while indication for cardiac stimulation was sick sinus syndrome in 27 $(56 \%)$ and atrioventricular block in $21(44 \%)$ in the $48 \mathrm{PM}$ patients.

\section{Significant lead-induced TR}

At baseline, some degree of TR (defined as grades 1-2) was present in 153 patients (64\%) patients and the distribution of TR grades in the whole patient population before (and after) lead implantation is summarised in figure 1. A significant worsening of TR was observed after lead implantation in the whole population (Wilcoxon $\mathrm{p}<0.001$ ) and, in particular, significant lead-induced TR was found in 91 (38\%) patients. Preimplantation clinical and echocardiographic characteristics of patients with significant lead-induced TR and no significant lead-induced TR are compared in table 1 . No significant differences were seen between the two groups, except for a trend (non-significant) towards more frequent atrial fibrillation, a higher prevalence of TR grade 1, and a higher RV fractional area change with a smaller RV end-diastolic area among patients with significant lead-induced TR.

\section{Impact of significant lead-induced TR on cardiac performance}

Echocardiographic changes after lead placement in patients with and without significant lead-induced TR are summarised in table 2. Similar changes over time in LVEF and diastolic function severity were seen between the two groups (table 2). Similar changes over time in LVEF, diastolic function and in mitral regurgitation severity were observed between the two groups (see interaction group and time $\mathrm{p}$ value in table 2 ). Although no significant changes over time in RV function (TAPSE and RV fractional area change) were seen in either group, RV size significantly increased over time only in patients with significant lead-induced TR. In addition, an enlargement of RA diameter was found in this group of patients. Finally, pulmonary

Table 3 Univariate and multivariate Cox regression survival analysis for the primary endpoint (all-cause mortality)

\begin{tabular}{|c|c|c|c|c|c|c|}
\hline \multirow[b]{2}{*}{ Variables } & \multicolumn{3}{|c|}{ Univariate analysis } & \multicolumn{3}{|c|}{ Multivariate analysis } \\
\hline & HR & $95 \% \mathrm{Cl}$ & $p$ Value & HR & $95 \% \mathrm{Cl}$ & $p$ Value \\
\hline Age, per year & 1.079 & 1.048 to 1.112 & $<0.001$ & 1.064 & 1.032 to 1.098 & $<0.001$ \\
\hline Male sex & 1.194 & 0.635 to 2.246 & 0.582 & & & \\
\hline Ischaemic aetiology & 1.684 & 0.963 to 2.944 & 0.068 & & & \\
\hline Atrial fibrillation & 1.373 & 0.823 to 2.290 & 0.224 & & & \\
\hline Diabetes & 1.705 & 0.963 to 3.018 & 0.067 & & & \\
\hline ICD system (versus PM) & 0.897 & 0.507 to 1.589 & 0.710 & & & \\
\hline Percentage of pacing, per $\%$ & 1.007 & 1.002 to 1.013 & 0.006 & 1.008 & 1.002 to 1.015 & 0.008 \\
\hline LVEDV, per mL & 1.005 & 1.002 to 1.009 & 0.001 & & & \\
\hline LVESV, per mL & 1.007 & 1.004 to 1.011 & $<0.001$ & & & \\
\hline LVEF, per \% & 0.968 & 0.946 to 0.990 & 0.005 & 0.973 & 0.947 to 0.999 & 0.041 \\
\hline Mitral regurgitation grade 0 (reference group) & & & 0.028 & & & 0.510 \\
\hline Mitral regurgitation grade 1 (vs reference group) & 0.449 & 0.219 to 0.922 & & 1.185 & 0.522 to 2.691 & \\
\hline Mitral regurgitation grade 2 (vs reference group) & 0.840 & 0.409 to 1.727 & & 1.445 & 0.626 to 3.336 & \\
\hline Mitral regurgitation grade 3 (vs reference group) & 1.815 & 0.737 to 4.468 & & 2.067 & 0.695 to 6.146 & \\
\hline Mitral regurgitation grade 4 (vs reference group) & 2.695 & 0.632 to 11.483 & & 2.634 & 0.662 to 10.488 & \\
\hline $\mathrm{RV}$ end-diastolic area, per $\mathrm{mm}^{2}$ & 1.069 & 1.025 to 1.114 & 0.002 & & & \\
\hline RV fractional area change, per $\%$ & 0.975 & 0.953 to 0.996 & 0.022 & & & \\
\hline TAPSE, per mm & 0.914 & 0.856 to 0.976 & 0.007 & 0.974 & 0.910 to 1.042 & 0.447 \\
\hline Right atrial diameter, per $\mathrm{mm}$ & 1.412 & 1.071 to 1.861 & 0.014 & & & \\
\hline Tricuspid annular diameter, per $\mathrm{mm}$ & 1.748 & 1.325 to 2.306 & $<0.001$ & & & \\
\hline sPAP, per $\mathrm{mm} \mathrm{Hg}$ & 1.046 & 1.029 to 1.063 & $<0.001$ & & & \\
\hline Significant lead-induced TR & 1.687 & 1.023 to 2.780 & 0.040 & 1.749 & 1.008 to 3.035 & 0.047 \\
\hline
\end{tabular}


pressures increased over time only in patients with lead-induced significant TR (from $33 \pm 11$ to $41 \pm 15 \mathrm{~mm} \mathrm{Hg}$ vs $33 \pm 12$ to 33 $\pm 10 \mathrm{~mm} \mathrm{Hg}$ (table 2).

\section{Impact of significant lead-induced TR on long-term prognosis}

The relation between significant lead-induced TR and the primary (all-cause mortality) and secondary (all-cause mortality and heart failure related events) endpoints was evaluated over a median long-term follow-up of 58 months (IQR 35-76 months) after repeated echocardiographic evaluation.

During the follow-up period, a total of 62 deaths $(26 \%)$ occurred. A higher all-cause death rate (primary endpoint) was seen in patients with significant lead-induced TR (log rank $\mathrm{p}=0.038$; figure $2 \mathrm{~A}$ ). In the univariate Cox proportional $\mathrm{HR}$ analysis, significant lead-induced TR reached a $\mathrm{HR}$ of 1.687 (95\% CI 1.023 to $2.780, p=0.040$ ) (table 3). After adjustment for the other clinical and echocardiographic characteristics, significant lead-induced TR was independently associated with survival (with adjusted $\mathrm{HR}=1.749$ (95\% CI 1.008 to 3.035$), p=0.047)$ together with age, percentage of pacing and LVEF.

Similarly, as shown in figure $2 \mathrm{~B}$, the secondary endpoint (combination of all-cause mortality and heart failure related events) was observed in 90 (38\%) patients. The secondary endpoint was more frequent in patients with lead-induced significant TR $(\log r a n k p=0.017)$. In the univariate analysis, significant lead-induced TR was associated with worse outcome with a $\mathrm{HR}=1.641(95 \% \mathrm{CI} 1.087$ to $2.480, \mathrm{p}=0.019)$ (table 4). In the multivariate model, significant lead-induced TR was independently associated with the occurrence of the secondary endpoint (adjusted HR=1.649, 95\% CI 1.043 to 2.599, $\mathrm{p}=0.032$ ) together with age, LVEF and mitral regurgitation.

The subgroup analysis in patients with baseline LVEF $<40 \%$ demonstrated that significant lead-induced TR was associated with poor survival free from the primary endpoint $(\mathrm{HR}=2.184$ (95\% CI 1.112 to 4.288 ), figure $3 \mathrm{~A}$ ) but not with survival free from the secondary endpoint $(\mathrm{HR}=1.428$ (95\% CI 0.832 to 2.451), figure 3B).

\section{DISCUSSION}

The main findings of this study include (1) significant lead-induced TR was observed in 38\% of patients $1-1.5$ years after placement of an RV lead; (2) significant lead-induced TR was associated with significant RV and right atrium enlargement, and with increased pulmonary pressures at follow-up and (3) significant lead-induced TR was independently associated with worse long-term clinical outcomes (all-cause mortality alone or combined with heart failure related events) after device implantation.

\section{Significant lead-induced TR}

The presence of a lead through the tricuspid valve apparatus has been suggested as one of the mechanisms of TR. However, only a few studies have described the incidence of lead-induced TR acutely after implantation and no data are available from the large randomised clinical trials on cardiac devices. ${ }^{35}{ }^{6} \mathrm{Kim}$ et al evaluated the presence of TR in 248 ICD or PM recipients shortly after implantation and found in 24\% of the patients an increase of TR by $>1$ grade. ${ }^{3}$ In addition, this increase in TR severity was more

Table 4 Univariate and multivariate Cox regression survival analysis for the secondary endpoint (all-cause mortality and heart failure related events)

\begin{tabular}{|c|c|c|c|c|c|c|}
\hline \multirow[b]{2}{*}{ Variables } & \multicolumn{3}{|c|}{ Univariate analysis } & \multicolumn{3}{|c|}{ Multivariate analysis } \\
\hline & HR & $95 \% \mathrm{Cl}$ & p Value & HR & $95 \% \mathrm{Cl}$ & p Value \\
\hline Age, per year & 1.039 & 1.018 to 1.061 & $<0.001$ & 1.026 & 1.003 to 1.050 & 0.030 \\
\hline Male sex & 0.908 & 0.557 to 1.481 & 0.699 & & & \\
\hline Ischaemic aetiology & 1.632 & 1.035 to 2.575 & 0.035 & 1.040 & 0.602 to 1.797 & 0.887 \\
\hline Atrial fibrillation & 1.058 & 0.683 to 1.639 & 0.801 & & & \\
\hline Diabetes & 1.277 & 0.777 to 2.098 & 0.335 & & & \\
\hline ICD device and lead & 1.283 & 0.764 to 2.155 & 0.347 & & & \\
\hline Percentage of pacing, per $\%$ & 1.003 & 0.999 to 1.008 & 0.157 & 1.001 & 0.996 to 1.007 & 0.636 \\
\hline LVEDV, per mL & 1.006 & 1.003 to 1.008 & $<0.001$ & & & \\
\hline LVESV, per mL & 1.008 & 1.005 to 1.011 & $<0.001$ & & & \\
\hline LVEF, per \% & 0.958 & 0.939 to 0.977 & $<0.001$ & 0.971 & 0.949 to 0.994 & 0.013 \\
\hline Mitral regurgitation grade 0 (reference group) & & & 0.011 & & & 0.014 \\
\hline Mitral regurgitation grade 1 (vs reference group) & 0.511 & 0.277 to 0.943 & & 1.175 & 0.618 to 2.235 & \\
\hline Mitral regurgitation grade 2 (vs reference group) & 1.042 & 0.572 to 1.896 & & 1.345 & 0.687 to 2.633 & \\
\hline Mitral regurgitation grade 3 (vs reference group) & 2.371 & 1.081 to 5.201 & & 2.659 & 1.126 to 6.276 & \\
\hline Mitral regurgitation grade 4 (vs reference group) & 0.915 & 0.124 to 6.732 & & 6.559 & 1.861 to 23.116 & \\
\hline $\mathrm{RV}$ end-diastolic area, per $\mathrm{mm}^{2}$ & 1.048 & 1.011 to 1.086 & 0.010 & & & \\
\hline RV fractional area change, per \% & 0.966 & 0.949 to 0.984 & $<0.001$ & & & \\
\hline TAPSE, per mm & 0.913 & 0.864 to 0.963 & 0.001 & 0.971 & 0.917 to 1.028 & 0.307 \\
\hline Right atrial diameter, per mm & 1.355 & 1.082 to 1.699 & 0.008 & & & \\
\hline Tricuspid annular diameter, per mm & 1.509 & 1.204 to 1.892 & $<0.001$ & & & \\
\hline sPAP, per mm Hg & 1.059 & 1.042 to 1.075 & $<0.001$ & & & \\
\hline Significant lead-induced TR & 1.641 & 1.087 to 2.480 & 0.019 & 1.649 & 1.043 to 2.599 & 0.032 \\
\hline
\end{tabular}




\section{A}

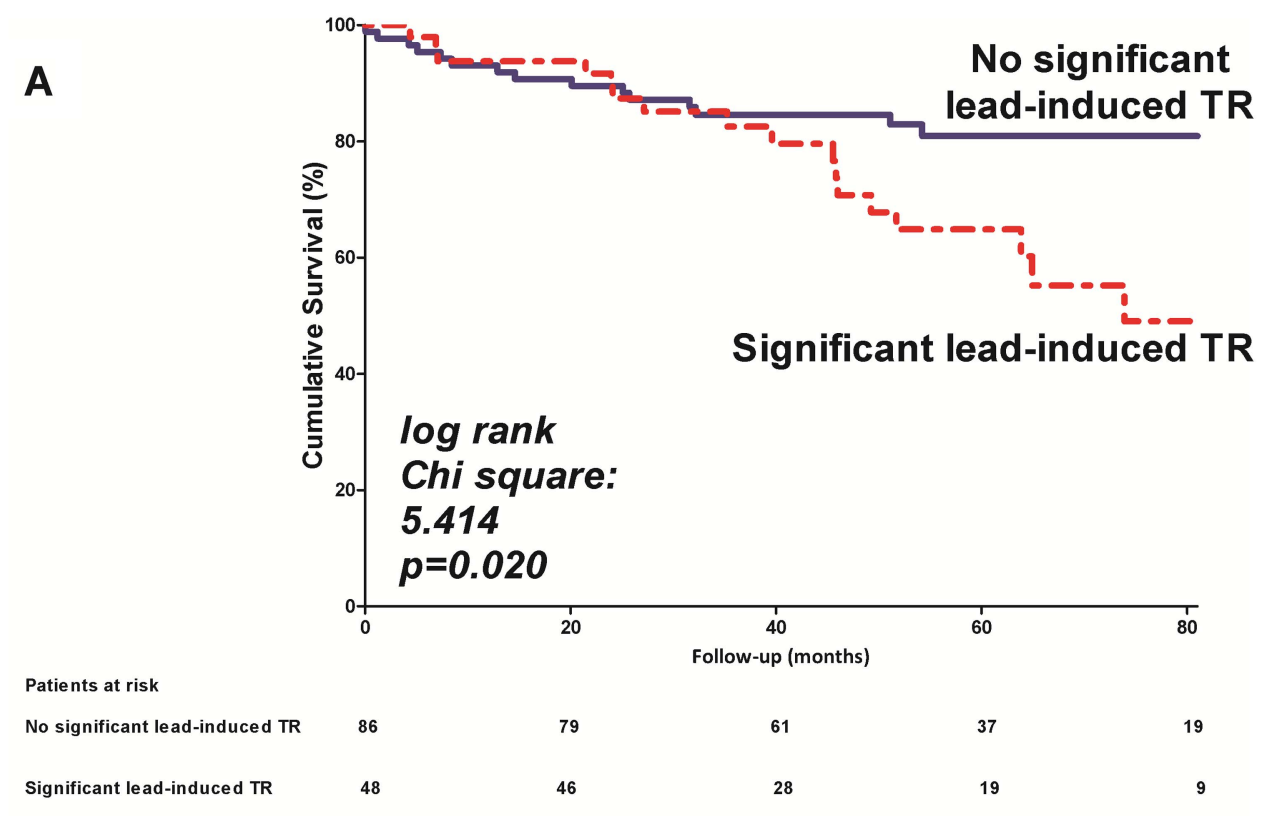

\section{No significant}

lead-induced TR

B

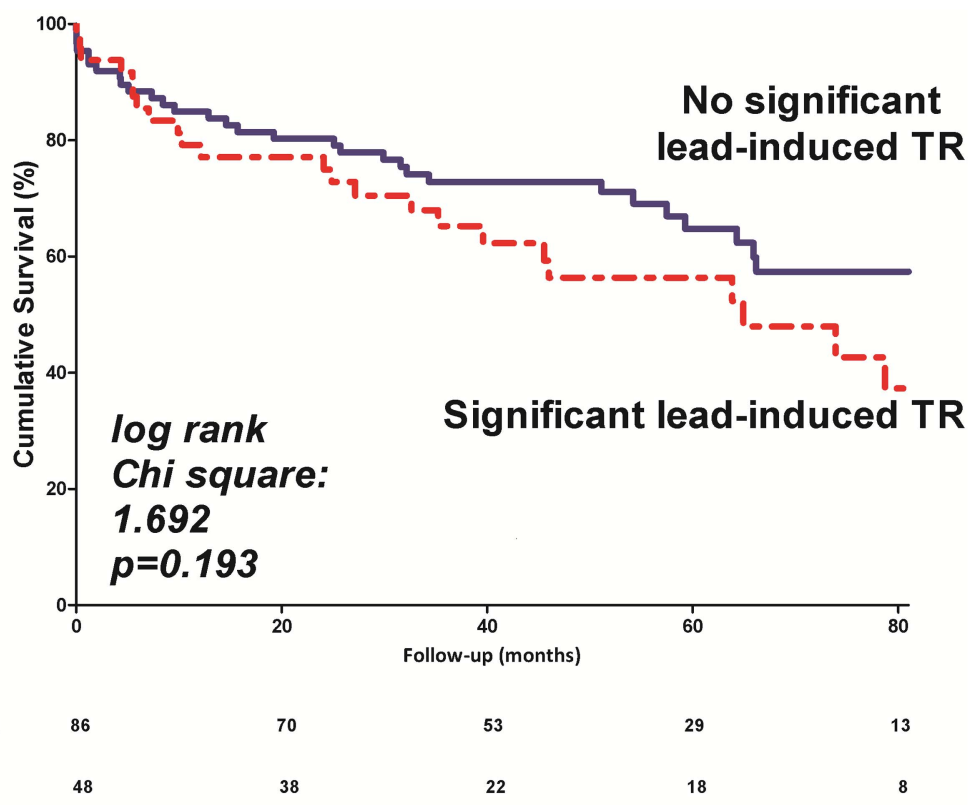

Figure 3 Subgroup analysis in patients with LVEF $<40 \%$ before device implantation. (A) Kaplan-Meier survival curves for the time to the primary endpoint (all-cause mortality) in patients with and without significant lead-induced tricuspid regurgitation (TR) with the follow-up onset at the second echocardiography. (B) Kaplan-Meier survival curves for the time to the secondary endpoint (all-cause mortality and heart failure related events) in patients with and without significant lead-induced TR with the follow-up onset at the second echocardiography.

pronounced among patients with no or trivial TR, while patients who already were grade 1-3 TR showed only modest worsening of TR. Our study, with longer term follow-up (1-1.5 years), reported a higher incidence of significant (grade $\geq 2$ ) TR progression (38\%), suggesting the additional role over time of chronic lead and valvular structural and functional alterations. Similarly, we observed a trend towards a higher incidence of significant lead-induced TR among patients with only trivial TR before implantation as compared with patients with significant TR. In patients with significant TR before device implantation, with dilated tricuspid annulus and/or of leaflet malcoaptation, the presence of an RV lead may not have significant additional impact on TR severity. In contrast, in patients with new-onset significant TR, an RV lead hampering proper leaflet coaptation seems to be an important pathophysiological factor.

The exact mechanism of development and progression of TR after cardiac device placement has not been fully elucidated and may result from the mechanical interference of the RV lead with the tricuspid valve ${ }^{3}{ }^{4}$ and/or from a direct effect on TR of RV pacing. ${ }^{20}$ In this study, no significant differences were seen in baseline clinical, echocardiography and device-related (ICD vs PM) characteristics among patients who did or did not develop significant TR after implantation, underlining the challenge of finding clear predisposing preimplantation parameters associated with lead-induced TR. These results suggest that significant lead-induced TR may result from 
progression of a pre-existing cardiac/valvular disease and also from interaction between the RV lead and tricuspid valve apparatus.

\section{Impact of significant lead-induced TR on cardiac performance}

This study showed that patients with significant lead-induced TR at follow-up had an increase in RV and RA dimensions and an increase in pulmonary pressures, while these parameters remained unchanged in patients without significant lead-induced TR. RV function, as assessed by TAPSE and RV fractional area change, did not change significantly at follow-up in patients with significant lead-induced TR, probably owing to the facilitated ejection in a low-resistance chamber (from the right ventricle to the right atrium).

The impairment of RV performance seen in patients with significant lead-induced TR is unlikely to be secondary to a worsening of LV performance. Indeed, the overall cohort showed a slight worsening of LV size and function at follow-up, as might be expected according to the natural history of heart failure or RV chronic pacing. However, no significant differences were noted in the changes over time in LV volumes and systolic and diastolic function among patients with or without significant lead-induced TR. Furthermore, the change over time in severity of mitral regurgitation was also similar among patients with or without significant lead-induced TR.

A potential explanation for increased pulmonary pressures, and therefore progression of TR and RV dilatation, might be the occurrence of multiple subclinical pulmonary emboli secondary to RV lead thrombus formation. Supple et al described a significantly higher increase in pulmonary pressure in patients with a mobile thrombus around the leads after cardiac device implantation as compared with patients without a mobile thrombus. ${ }^{21}$ However, the increase in pulmonary pressures did not reach the cut-off value proposed by current guidelines to define pulmonary hypertension $(>50 \mathrm{~mm} \mathrm{Hg})$ and therefore the diagnosis of pulmonary hypertension is not definitive in the absence of other echocardiographic parameters. ${ }^{18}$ This suggests that the development of significant TR after lead implantation plays a primary role and is one of the major determinants of the changes in RV performance.

\section{Impact of significant lead-induced TR on long-term prognosis}

Although the presence of significant TR, regardless of aetiology, is a well-known prognostic factor, data on long-term outcome in patients with significant TR after RV lead implantation have not been reported. ${ }^{2}$ Our study demonstrated the independent association between significant lead-induced TR and all-cause mortality (combined with heart failure related events or not). Other variables independently associated with long-term outcomes (primary and secondary) were age, LVEF, percentage of RV pacing and significant mitral regurgitation, which are all known determinants of the development of heart failure and increased mortality. In particular, subanalysis according to the baseline LVEF showed that significant lead-induced TR in patients with a depressed LVEF $(<40 \%)$ at baseline was associated with poor prognosis. RV pacing has been previously shown to be associated with an increased risk of LV (progressive) dysfunction and heart failure events. ${ }^{22-24}$ This detrimental effect of RV pacing might be mediated by induction of LV dyssynchrony ${ }^{24}{ }^{25}$ but also by a direct negative effect on the severity of TR, as suggested by Vaturi et al. ${ }^{20}$ However, analyses of large trials showed that implantation of an ICD, even with minimal percentage of RV pacing, was associated with an increased risk of hospitalisations for congestive heart failure and death as compared with controls. ${ }^{26}$ The exact reason for this increased risk of heart failure events has not yet been elucidated but, considering the results of our study, might also be explained by lead-induced TR. Indeed, even modest grades of TR were associated with an increased risk of all-cause mortality.

These findings emphasise the importance of echocardiographic surveillance of ICD and PM recipients, enabling anticipation of the development of heart failure. Although lead repositioning or extraction might be an option only in the shortterm after implantation, other therapeutic options, such as upgrade to a biventricular pacemaker, optimisation of heart failure drugs or a surgical procedure on the tricuspid valve, might be considered in patients with significant TR and/or worsening LV function.

Several limitations of this study should be mentioned. First, the exact mechanism of lead-induced TR could not be confirmed in all patients. In addition, it remains unclear whether progression of RV remodelling is the cause or the consequence of significant TR. Moreover, the time interval between echocardiographic evaluations of 1-1.5 years was chosen to ensure identification of both acute and long-term occurrence of significant TR, but might still have underestimated the incidence of this complication, particularly because patients who died within 1 year after implantation were excluded. The mode of death was not systematically available and the impact of significant lead-induced TR on cardiovascular mortality could not be assessed. Finally, prospective studies with larger patient groups and longer follow-up are needed.

\section{Key messages}

What is already known about this subject?

- Trivial tricuspid regurgitation (TR) is a common echocardiographic finding in healthy individuals. However, significant TR (grade $\geq 2$ ) has been shown to be associated with poor prognosis, regardless of the underlying cardiac pathology.

- Placement of an RV (trans-tricuspid) lead has been suggested as a cause of TR. However, the incidence of lead-induced TR, time course and, more importantly, data on the impact of significant TR on cardiac performance and clinical outcome remain unknown.

What does this study add?

- This study evaluated for the first time the impact of significant lead-induced TR on cardiac performance and on the long-term prognosis. A $>35 \%$ incidence of significant TR was seen at follow-up after implantation of an RV lead. Furthermore, significant lead-induced TR was associated with a significant RV and right atrium enlargement and, importantly, with a higher incidence of long-term mortality and heart failure events.

How might this impact on clinical practice?

- The findings of this study suggest the importance of an echocardiographic follow-up in implantable cardioverter-defibrillator and pacemaker recipients in order to optimise patient management, including optimisation of heart failure treatment and eventually, tricuspid valve surgery. 
In conclusion, a significantly increased incidence of TR was seen at follow-up after implantation of an RV lead in more than 35\% of ICD and PM recipients. A significant lead-induced TR was associated with an impaired RV performance and with a higher incidence of long-term mortality and heart failure events. These findings suggest the importance of echocardiographic follow-up in these patients in order to optimise patient management.

Acknowledgements Department of Cardiology of Leiden University Medical Centre received unrestricted research grants from Medtronic, Biotronik, Boston Scientific, Lantheus Medical Imaging, St Jude Medical, Edwards Life Sciences and GE Healthcare. VD received consulting fees from St Jude Medical and Medtronic.

Contributors $U H, D A, J T, V D, J J B$ and NAM were responsible for study design. $\mathrm{UH}, \mathrm{DA}, \mathrm{JT}, \mathrm{ERH}$ and ETvdV participated in data collection. UH, RW, DA, VD and NAM analysed the data and drafted the manuscript. JJB, MJS VD and NAM critically revised the manuscript. All authors approved the final version of the submitted manuscript.

\section{Competing interests None.}

Provenance and peer review Not commissioned; externally peer reviewed.

\section{REFERENCES}

1 Singh JP, Evans JC, Levy D, et al. Prevalence and clinical determinants of mitral, tricuspid, and aortic regurgitation (the Framingham Heart Study). Am J Cardiol 1999:83:897-902.

2 Nath J, Foster E, Heidenreich PA. Impact of tricuspid regurgitation on long-term survival. J Am Coll Cardiol 2004:43:405-9.

3 Kim JB, Spevack DM, Tunick PA, et al. The effect of transvenous pacemaker and implantable cardioverter defibrillator lead placement on tricuspid valve function: an observational study. J Am Soc Echocardiogr 2008;21:284-7.

4 Lin G, Nishimura RA, Connolly HM, et al. Severe symptomatic tricuspid valve regurgitation due to permanent pacemaker or implantable cardioverter-defibrillator leads. J Am Coll Cardiol 2005;45:1672-5.

5 Klutstein M, Balkin J, Butnaru A, et al. Tricuspid incompetence following permanent pacemaker implantation. Pacing Clin Electrophysiol 2009;32(Suppl 1):S135-7.

6 Kucukarslan N, Kirilmaz A, Ulusoy E, et al. Tricuspid insufficiency does not increase early after permanent implantation of pacemaker leads. J Card Surg 2006;21:391-4.

7 Paniagua D, Aldrich HR, Lieberman EH, et al. Increased prevalence of significant tricuspid regurgitation in patients with transvenous pacemakers leads. Am J Cardiol 1998:82:1130-2, A9.

8 Alizadeh A, Sanati HR, Haji-Karimi M, et al. Induction and aggravation of atrioventricular valve regurgitation in the course of chronic right ventricular apical pacing. Europace 2011;13:1587-90.

9 Sakai M, Ohkawa S, Ueda K, et al. Tricuspid regurgitation induced by transvenous right ventricular pacing: echocardiographic and pathological observations. J Cardiol 1987; 17:311-20.

10 Uslan DZ, Tleyjeh IM, Baddour LM, et al. Temporal trends in permanent pacemaker implantation: a population-based study. Am Heart J 2008;155:896-903.
11 Santangeli P, Di Biase L, Dello Russo A, et al. Meta-analysis: age and effectiveness of prophylactic implantable cardioverter-defibrillators. Ann Intern Med 2010;153:592-9.

12 Lin G, Meverden RA, Hodge DO, et al. Age and gender trends in implantable cardioverter defibrillator utilization: a population based study. J Interv Card Electrophysiol 2008;22:65-70.

13 Tracy CM, Epstein AE, Darbar D, et al. 2012 ACCF/AHA/HRS focused update of the 2008 guidelines for device-based therapy of cardiac rhythm abnormalities: a report of the American College of Cardiology Foundation/American Heart Association Task Force on Practice Guidelines. Heart Rhythm 2012;9:1737-53.

14 Moss AJ, Hall WJ, Cannom DS, et al. Improved survival with an implanted defibrillator in patients with coronary disease at high risk for ventricular arrhythmia. Multicenter automatic defibrillator implantation trial investigators. N Eng/ J Med 1996:335:1933-40.

15 Greenberg H, Case RB, Moss AJ, et al. Analysis of mortality events in the Multicenter Automatic Defibrillator Implantation Trial (MADIT-II). J Am Coll Cardiol 2004;43:1459-65.

16 Lancellotti P, Moura L, Pierard LA, et al. European Association of Echocardiography recommendations for the assessment of valvular regurgitation. Part 2: mitral and tricuspid regurgitation (native valve disease). Eur J Echocardiogr 2010;11:307-32.

17 Lang RM, Bierig M, Devereux RB, et al. Recommendations for chamber quantification. Eur J Echocardiogr 2006;7:79-108

18 Galie N, Hoeper MM, Humbert M, et al. Guidelines for the diagnosis and treatment of pulmonary hypertension: the Task Force for the Diagnosis and Treatment of Pulmonary Hypertension of the European Society of Cardiology (ESC) and the European Respiratory Society (ERS), endorsed by the International Society of Heart and Lung Transplantation (ISHLT). Eur Heart J 2009;30:2493-537.

19 Rudski LG, Lai WW, Afilalo J, et al. Guidelines for the echocardiographic assessment of the right heart in adults: a report from the American Society of Echocardiography endorsed by the European Association of Echocardiography, a registered branch of the European Society of Cardiology, and the Canadian Society of Echocardiography. J Am Soc Echocardiogr 2010;23:685-713; quiz 86-8.

20 Vaturi M, Kusniec J, Shapira Y, et al. Right ventricular pacing increases tricuspid regurgitation grade regardless of the mechanical interference to the valve by the electrode. Eur J Echocardiogr 2010;11:550-3.

21 Supple GE, Ren JF, Zado ES, et al. Mobile thrombus on device leads in patients undergoing ablation: identification, incidence, location, and association with increased pulmonary artery systolic pressure. Circulation 2011;124:772-8.

22 Lamas GA, Lee KL, Sweeney MO, et al. Ventricular pacing or dual-chamber pacing for sinus-node dysfunction. N Engl J Med 2002;346:1854-62.

23 Wilkoff BL, Cook JR, Epstein AE, et al. Dual-chamber pacing or ventricular backup pacing in patients with an implantable defibrillator: the Dual Chamber and VVI Implantable Defibrillator (DAVID) Trial. JAMA 2002;288:3115-23.

24 Sweeney MO, Hellkamp AS, Ellenbogen KA, et al. Adverse effect of ventricular pacing on heart failure and atrial fibrillation among patients with normal baseline QRS duration in a clinical trial of pacemaker therapy for sinus node dysfunction. Circulation 2003;107:2932-7.

25 Delgado V, Tops LF, Trines SA, et al. Acute effects of right ventricular apical pacing on left ventricular synchrony and mechanics. Circ Arrhythm Electrophysiol 2009;2:135-45

26 Goldenberg I, Moss AJ, Hall WJ, et al. Causes and consequences of heart failure after prophylactic implantation of a defibrillator in the multicenter automatic defibrillator implantation trial II. Circulation 2006;113:2810-17. 